\title{
Effet du climat tropical humide sur la température rectale et les fréquences respiratoire et cardiaque des taurillons Créoles en Guadeloupe (Antilles françaises)
}

\author{
P. BERBIGIER
}

I.N.R.A., Station de Bioclimatologie et Station de Recherches zootechniques

Domaine Duclos, 97170 Petit-Bourg (Guadeloupe)

\begin{abstract}
Résumé
Seize taurillons Créoles sont soumis à deux traitements climatiques, en plein air et dans une étable ouverte, pourvue d'un toit en tôle, et à deux régimes alimentaires, un régime "concentré » $(80$ p. 100 d'aliment concentré et 20 p. 100 de fourrage) et un régime "fourrage» (respectivement 32 p. 100 d'aliment concentré et 68 p. 100 de fourrage), selon un schéma factoriel $2^{2}$. Entre 11 et $13 \mathrm{~h}$, la température rectale, les fréquences respiratoire et cardiaque, ainsi que les variables climatiques et la température du "corps noir» sont mesurées, au cours de 44 journées d'expérience. L'effet des traitements est analysé par analyse de variance, celui des variables climatiques par régression multiple.

La fréquence respiratoire est la plus dépendante des variables climatiques, bien que la différence plein air-abri ne soit pas significative. La température rectale, pour laquelle cette différence atteint le seuil $\mathbf{P}<0,01$, est pourtant peu dépendante des variables climatiques, de même que la fréquence cardiaque. Dans tous les cas, l'effet du climat est plus sensible en plein air, et particulièrement pour le régime "fourrage», dont la production de chaleur estimée est supérieure à celle du régime "concentré ».

Les taurillons Créoles doivent mettre en jeu des mécanismes de thermolyse par évaporation (fréquence respiratoire) pour équilibrer leur bilan thermique. Néanmoins ils ne subissent pas de stress thermique (température rectale modérée). Ils semblent donc bien adaptés au climat tropical antillais.
\end{abstract}

\section{Introduction}

Dans un précédent article (BERBigier, 1983 a), nous avons montré que la consommation d'aliment et la croissance pondérale des taurillons Créoles en Guadeloupe étaient peu affectées par le climat tropical, même en plein soleil. Le but du présent travail est d'étudier l'effet de ce climat sur quelques paramètres physiologiques qui caractérisent la sensibilité à la chaleur : la fréquence respiratoire, la température rectale et la fréquence cardiaque. Deux situations climatiques très contrastées (animaux au soleil et animaux à l'ombre) ainsi que deux régimes alimentaires sont comparés. 
Mount (1974) résume l'ensemble des phénomènes qui tendent à rétablir l'équilibre thermique lorsque la chaleur ambiante dépasse la zone de confort thermique d'un animal. Il montre que les premières réactions de celui-ci sont une augmentation de la transpiration et de l'évaporation respiratoire, donc de la fréquence respiratoire. La température rectale, en théorie tout du moins, ne s'élève que lorsque les possibilités de thermorégulation sont épuisées. En fait, chez les bovins, on constate un accroissement parallèle de la fréquence respiratoire et de la température rectale, même lorsque la contrainte thermique est modérée et sans que la température rectale dépasse les limites normales relatives à un animal bien portant (BERBIGIER, 1982). Quant à la fréquence cardiaque, elle augmente, en réponse à la chaleur, parallèlement à l'augmentation du métabolisme qui résulte du stress thermique (INGRAM \& MounT, 1975). Elle ne doit donc pas être affectée par une contrainte climatique légère.

Ainsi, par l'étude de ces trois variables, il est possible de définir l'état physiologique (neutralité thermique, thermorégulation ou stress thermique) des taurillons Créoles soumis à l'action du climat tropical, et de juger objectivement de leur adaptation thermique à celui-ci.

\section{Matériel et méthodes}

16 taurillons Créoles d'un poids initial de $167 \pm 20 \mathrm{~kg}$ et âgés de $346 \pm 98$ jours sont répartis en 4 blocs en fonction du poics, de l'âge, et du gain de poids réalisé au cours de la période préexpérimentale d'une durée de 50 jours. Les animaux de chaque bloc sont ensuite affectés à 4 traitements suivant un schéma factoriel $2^{2}$ correspondant à deux situations climatiques et à deux régimes alimentaires. Dans la première situation climatique, les animaux sont placés à l'attache dans des stalles non protégées des intempéries, dans la seconde ils sont abrités sous un toit en tôle (situations «sans » et «avec abri »). Le premier régime ("concentré ») est composé, pour 80 p. 100 de la matière sèche, d'aliment concentré, et pour 20 p. 100 de fourrage vert de prairie naturelle («petit foin »), le deuxième («fourrage») comporte les mêmes composants dans des proportions de 32 et 68 p. 100 . Les mesures ne commencent que 65 jours après le début des traitements, le changement de régime ayant perturbé la croissance des taurillons. Les caractéristiques des animaux, des rations et des situations climatiques sont décrites en détail par allleurs (BERBIGIER, 1983 a).

La production de chaleur de chaque animal est calculée à partir de l'estimation de l'énergie métabolisable (EM) ingérée et de celle de l'énergie fixée, selon la méthode déjà utilisée par Berbigier (1983 b). Pour les régimes «fourrage», il est tenu compte de la diminution de la teneur en EM due aux phénomènes d'associativité (I.N.R.A., 1978).

Deux fois par semaine, entre 11 et $13 \mathrm{~h}$ (heure locale), la température rectale, la fréquence respiratoire (par comptage des battements de flanc) et le pouls (pris à la queue) sont mesurés pour chaque animal. Simultanément, la température ambiante (Ta), celle du thermomètre humide $(\mathrm{Th})$ et celle du «corps noir » $(\mathrm{Tb}$, boule de $10 \mathrm{~cm}$ de diamètre) sont relevées; au total, 44 journées de mesures sont utilisables, dont 31 pour lesquelles $\mathrm{Tb}$ est connue. La vitesse moyenne du vent pendant les mesures (V) est donnée par deux anémomètres à coupelle, de seuil de démarrage $0,8 \mathrm{~m} / \mathrm{s}$, placés au 
niveau des animaux à l'intérieur et à l'extérieur. Les rayonnements globaux pendant les mesures $(R)$ et cumulés depuis le matin jusquà l'heure de celles-ci sont enregistrés par une thermopile et un dispositif intégrateur. La pluviométrie entre $7 \mathrm{~h}$ du matin et la fin des mesures (PIm) ainsi que celle de la veille (Plv, de $7 \mathrm{~h}$ du matin la veille à $7 \mathrm{~h}$ le jour des mesures) sont lues au pluviomètre. La température minimale de la nuit précédente (Tmin) est enregistrée sur un thermographe, dans un abri météorologique situé à proximité. L'humidité maximale de la nuit, pratiquement constante, n'est pas prise en compte.

L'albedo (réflectivité pour le rayonnement solaire) des pelages est mesurée deux fois, au début et à la fin de la période expérimentale, à l'aide d'une thermopile tournée alternativement vers le pelage et vers le ciel.

Deux types d'analyse statistique sont effectuées :

- une analyse de variance portant sur les moyennes, calculées pour chaque animal, et relatives à toutes les journées de mesure, de la température rectale et des fréquences respiratoire et cardiaque; elle permet de tester l'effet moyen des traitements sur l'ensemble de la période expérimentale (SNEdEcor \& Cochran, 1971);

- une analyse en régression linéaire multiple progressive (Dagnelie, 1975) qui permet de tester jour par jour les effets des variables climatiques, du poids vif et de la quantité d'énergie métabolisable (EM) ingérée sur les moyennes par traitement de la température rectale, de la fréquence respiratoire et de la fréquence cardiaque. La quantité d'EM ingérée est prise à la place de la production de chaleur, qui, calculée à l'aide du gain de poids (BERBIGIER, 1983 b), ne peut être connue à l'échelle de la journée. La température du thermomètre humide Th est remplacée par celle du point de rosée, calculée à partir de $\mathrm{Ta}$ et $\mathrm{Th}$, qui est plus caractéristique de l'humidité de l'air. Les variables explicatives les moins significatives sont successivement éliminées pour obtenir un modèle minimum.

De plus, l'effet de la «température du corps noir » sur la température rectale, la fréquence respiratoire et la fréquence cardiaque est étudié au moyen d'une analyse en régression triple linéaire comprenant aussi, comme variables explicatives, le point de rosée et le poids des animaux : «la température du corps noir» est supposée intégrer l'effet des autres variables climatiques.

\section{Résultats}

\section{A. Conditions climatiques}

Sur les 44 journées d'expérience, les moyennes ( \pm écart-type) des variables climatiques utilisées dans les analyses en régression multiple sont :

- température de l'air pendant la mesure : $28,4 \pm 1,5^{\circ} \mathrm{C}$ (valeurs extrêmes 31,2 et $23,5^{\circ} \mathrm{C}$ ) ;

- température minimale de la nuit précédente : $23,1 \pm 1,6^{\circ} \mathrm{C}$ (valeurs extrêmes 25,6 et $\left.18,5^{\circ} \mathrm{C}\right)$;

- point de rosée pendant la mesure : $22,3 \pm 1,4^{\circ} \mathrm{C}$; 
- rayonnement global pendant la mesure : $247 \pm 71 \mathrm{~J} \cdot \mathrm{cm}^{-2} \cdot \mathrm{h}^{-1}$ (valeurs extrêmes 354 et $63 \mathrm{~J} \cdot \mathrm{cm}^{-2} \cdot \mathrm{h}^{-1}$ ); J. $\mathrm{cm}^{-2}$;

- rayonnement global cumulé de laube à la fin des mesures : $808 \pm 278$

- vitesse du vent à l'extérieur pendant la mesure : $2,56 \pm 0,80 \mathrm{~m} \cdot \mathrm{s}^{-1}$;

- vitesse du vent à l'intérieur pendant la mesure : $2,02 \pm 0,75 \mathrm{~m} \cdot \mathrm{s}^{-1}$;

— pluie de $7 \mathrm{~h}$ du matin à la fin des mesures : $1,9 \pm 8,2 \mathrm{~mm}$;

— pluie de la veille du jour des mesures : 3,5 $\pm 11,5 \mathrm{~mm}$.

La température du «corps noir » (31 journées d'expérience) pendant les mesures est de $35,5 \pm 3,9$ "C à l'extérieur et de $29,2 \pm 1,6^{\circ} \mathrm{C}$ à l'intérieur.

\section{B. Production de chaleur}

Sur l'ensemble de la période expérimentale, la production de chaleur moyenne par traitement, ramenée à l'unité de surface cutanée, est, pour les régimes « concentré », de $120 \mathrm{Wm}^{-2}$ sans abri et de $151 \mathrm{Wm}^{-2}$ avec abri; pour les régimes "fourrage », elle est de $136 \mathrm{Wm}^{-2}$ sans abri et $148 \mathrm{Wm}^{-2}$ avec abri. L'analyse de variance montre un effet significatif $(\mathrm{P}<0,05)$ de la situation climatique, mais pas du régime. L'interaction n'atteint pas le niveau $P<0,05$. Ces résultats s'expliquent par l'effet dépressif de la situation «sans abri» sur la consommation, en particulier pour le régime «concentré», alors que le gain de poids n'est pas affecté par l'absence d'abri (BERBIGIER, 1983 a).

\section{Albedo du pelage}

Les albedos moyennes pour les animaux exposés au soleil sont de $0,192 \pm 0,026$ (régime «concentré», moyenne \pm - écart-type) et de $0,171 \pm 0,050$ (régime «fourrage »). La différence entre les deux groupes d'animaux n'est pas significative. L'albedo n'intervient pas dans les échanges thermiques des animaux non exposés au soleil et n'a pas été mesurée.

\section{Valeurs moyennes de la température rectale et des fréquences respiratoire et cardiaque sur la période expérimentale (tabl. 1)}

Aucune interaction entre les effets climatiques et nutritionnels n'est significative au seuil 5 p. 100.

La température rectale est plus élevée en l'absence d'abri qu'avec abri (moyennes tous régimes confondus : 39,00 " $\mathrm{C}$ contre 38,78 " $\mathrm{C}, \mathrm{P}<0,01$ ), et pour les régimes «fourrage » que pour les régimes « concentré » (moyenne toutes situations climatiques confondues : 38,98 " $\mathrm{C}$ contre $38,81^{\circ} \mathrm{C}, \mathrm{P}<0,05$ ). Il n'y a aucun effet significatif au seuil 5 p. 100 sur les fréquences respiratoire et cardiaque, dont les moyennes tous traitements confondus sont respectivement 27,5 respirations/mn et 92,3 battements/mn. Cependant, la fréquence respiratoire semble légèrement plus élevée sans abri qu'avec abri (moyenne tous régimes confondus : $29,8 \mathrm{resp} / \mathrm{mn}$ contre $25,3 \mathrm{resp} / \mathrm{mn}, \mathbf{P}<0,10$ ). 


\section{TABleaU 1}

Valeurs moyennes sur la période expérimentale, de la température rectale, de la fréquence respiratoire et de la fréquence cardiaque.

Mean values during the experimental period of rectal temperature, respiratory rhythm and heart beat rate.

\begin{tabular}{|c|c|c|c|c|}
\hline \multirow{2}{*}{$\begin{array}{l}\begin{array}{l}\text { Situation climatique } \ldots \\
\text { Climatic situation }\end{array} \\
\text { Régime } \ldots \ldots \ldots \ldots \ldots \\
\text { Diet }\end{array}$} & \multicolumn{2}{|c|}{$\begin{array}{c}\text { «Sans abri } \\
\text { «Without shelter }\end{array}$} & \multicolumn{2}{|c|}{$\begin{array}{l}\text { «Avec abri » } \\
\text { «With shelter» }\end{array}$} \\
\hline & $\begin{array}{l}\text { «Concentré » } \\
\text { «Concentrate» }\end{array}$ & $\begin{array}{l}\text { «Fourrage» } \\
\text { «Forage» }\end{array}$ & $\begin{array}{l}\text { «Concentré» } \\
\text { «Concentrate» }\end{array}$ & $\begin{array}{c}\text { «Fourrage» } \\
\text { «Forage }\end{array}$ \\
\hline $\begin{array}{l}\text { Température rectale . } \\
\text { Rectal temperature } \\
\left({ }^{\circ} \mathrm{C}\right)\end{array}$ & 38,89 & 39,12 & 38,72 & 38,83 \\
\hline $\begin{array}{l}\text { Fréquence respiratoire } \\
\text { Respiratory rhythm } \\
\text { (resp/min) }\end{array}$ & 31,9 & 27,6 & 26,7 & 23,9 \\
\hline $\begin{array}{l}\text { Fréquence cardiaque .. } \\
\text { (battements } / \mathrm{mn} \text { ) } \\
\text { Heart beat rate } \\
\text { (beat } / \mathrm{mn} \text { ) }\end{array}$ & 94,4 & 94,3 & 94,1 & 86,2 \\
\hline
\end{tabular}

E. Relations entre la température rectale, les fréquences respiratoire et cardiaque, et les variables climatiques et le poids (tabl. 2)

L'énergie métabolisable ingérée journalière n'a eu aucun cas d'effet significatif et ne figure pas sur le tableau 2. Il en est de même pour le rayonnement cumulé depuis le lever du soleil.

\section{Température rectale}

Elle dépend très peu des variables climatiques. Le seul effet climatique significatif est celui du rayonnement global $R$ sur le traitement «fourrage sans abri $»\left(+0,23{ }^{\circ} \mathrm{C}\right.$ pour une augmentation de $100 \mathrm{~J} \mathrm{.} \mathrm{cm}^{-2} \cdot \mathrm{h}^{-1}$, c'est-à-dire $280 \mathrm{Wm}^{-2}$ ). En revanche, pour tous les traitements, elle diminue significativement lorsque le poids des animaux augmente, phénomène déjà observé précédemment (BERBigier, 1983 b). Le coefficient de détermination relatif aux différents modèles varie de 0,21 à 0,48 .

\section{Fréquence respiratoire}

Elle est plus sensible aux variables climatiques. Parmi les taurillons « sans abri », ceux qui sont soumis au régime "concentré » ne sont sensibles qu'à la température ambiante $\left(+3,5\right.$ respirations $/ \mathrm{mn}$ lorsque Ta augmente de $\left.1^{\circ} \mathrm{C}\right)$ et au vent $(-3,0$ respirations/mn lorsque $\mathrm{V}$ augmente de $1 \mathrm{~ms}^{-1}$ ); ceux qui sont soumis au régime 


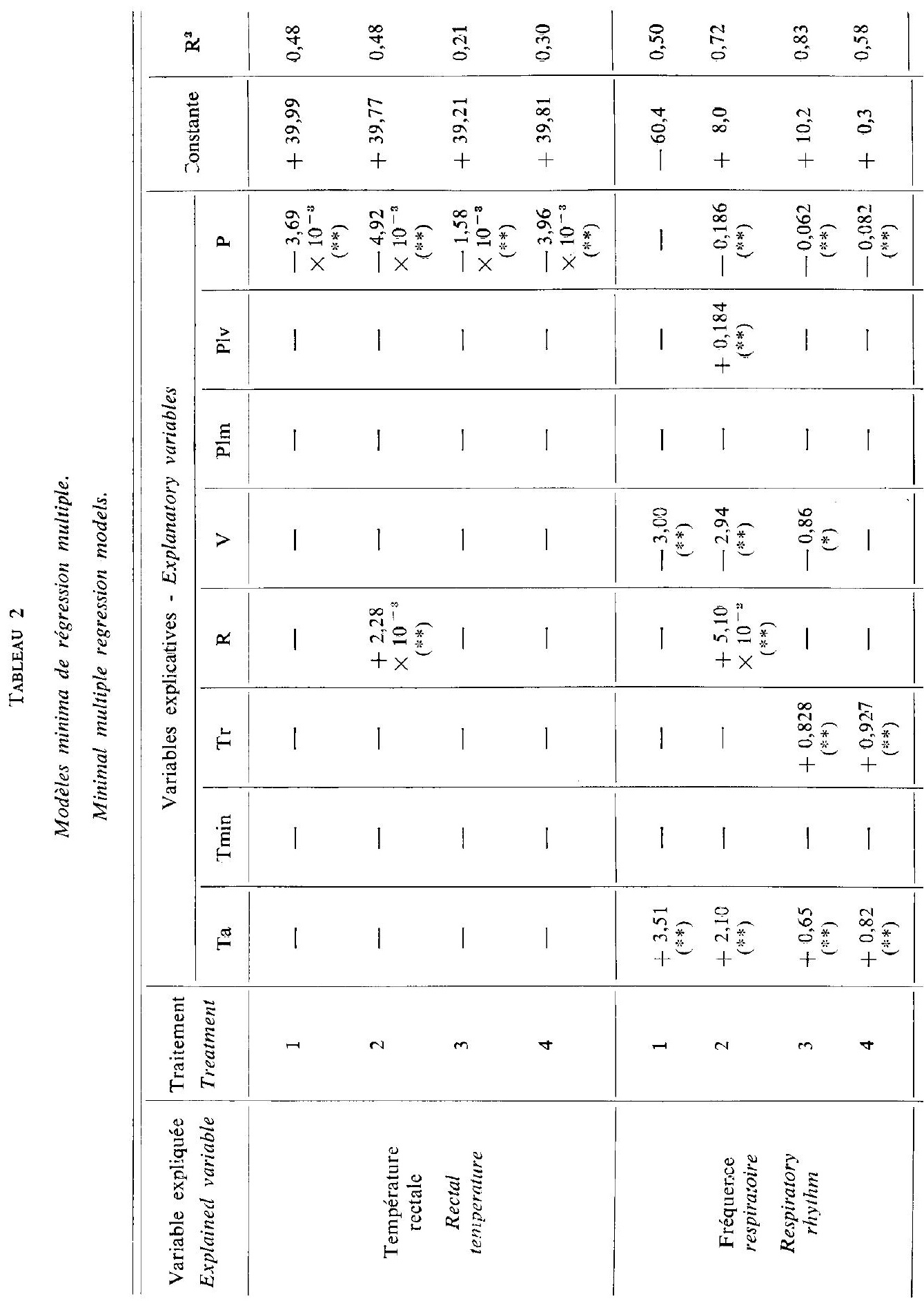




\begin{tabular}{|c|c|c|c|}
\hline $\bar{\sigma}$ & $\begin{array}{l}0 \\
0 \\
0\end{array}$ & 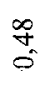 & $\begin{array}{l}\text { q } \\
0\end{array}$ \\
\hline $\begin{array}{l}5 \\
\text { ले } \\
+\end{array}$ & $\stackrel{\infty}{\subseteq}$ & $\begin{array}{l}\text { त्र } \\
\text { ปै } \\
+\end{array}$ & $\overrightarrow{0}$ \\
\hline 1 & 1 & 1 & 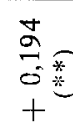 \\
\hline 1 & 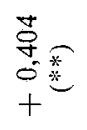 & 1 & 1 \\
\hline \begin{tabular}{l}
$\stackrel{a}{2}$ \\
\multirow{2}{0}{$\approx$} \\
+
\end{tabular} & I & 1 & 1 \\
\hline
\end{tabular}

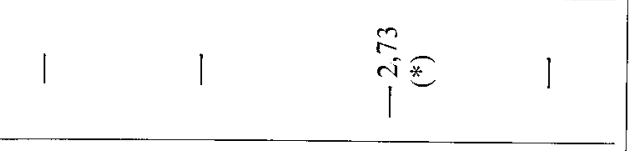

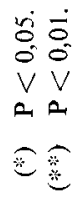

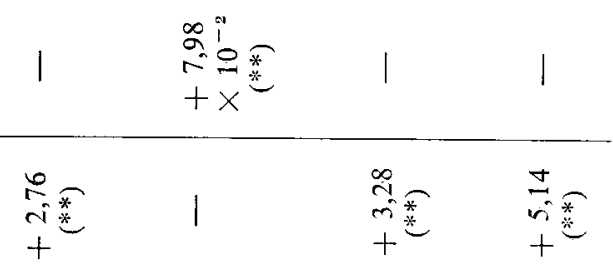

$\stackrel{+\infty}{n_{n}^{-\pi}}$<smiles>C1CCCC1</smiles>

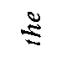

.

.


«fourrage » réagissent non seulement à $\mathrm{Ta}\left(+2,1 \mathrm{resp} / \mathrm{mn}\right.$ par $\left.{ }^{\circ} \mathrm{C}\right)$ et à $\mathrm{V}(-2,9$ $\mathrm{resp} / \mathrm{mn}$ par $\left.\mathrm{ms}^{-1}\right)$, mais aussi au rayonnement global $(+5,1 \mathrm{resp} / \mathrm{mn}$ lorsque $\mathrm{R}$ augmente de $\left.100 \mathrm{~J} \cdot \mathrm{cm}^{-2} \cdot \mathrm{h}^{-1}\right)$ et à la pluie du jour précédent $(+0,18 \mathrm{resp} / \mathrm{mn}$ par $\mathrm{mm}$ de pluie).

Avec abri, l'effet du rayonnement global disparaît et celui de la température de point de rosée apparaît dans les deux cas (respectivement $+0,83$ et $+0,94 \mathrm{resp} / \mathrm{mn}$ par ${ }^{\circ} \mathrm{C}$ pour les régimes «concentré » et «fourrage»). Celui de la température ambiante demeure (respectivement $+0,65$ et $+0,82 \mathrm{resp} / \mathrm{mn}$ par ${ }^{\circ} \mathrm{C}$ ). Le rôle du vent n'est significatif que pour le régime «concentré » $\left(-0,86 \mathrm{resp} / \mathrm{mn}\right.$ par $\left.\mathrm{ms}^{-1}\right)$.

La fréquence respiratoire diminue avec le poids des animaux pour tous les traitements sauf le traitement «concentré sans abri », en accord avec nos résultats précédents (Berbigier, 1983 b). Les coefficients de détermination sont, sans abri, de 0,50 et 0,72 pour les régimes "concentré " et "fourrage», et, avec abri, de 0,83 et 0,58 pour ces mêmes régimes.

\section{Fréquence cardiaque}

Elle est fortement reliée, dans trois traitements sur quatre, à la température de point de rosée $\left(+2,8\right.$ pulsations $/ \mathrm{mn}$ par ${ }^{\circ} \mathrm{C}$ pour le traitement «concentré sans abri », et, avec abri, respectivement $+3,3$ et $+5,1$ pulsations $/ \mathrm{mn}$ pour les régimes «concentré » et «fourrage»). Les autres effets climatiques observés sur ces trois traitements sont peu nombreux et peu significatifs : effet positif de la pluie du matin sur les animaux sans abri au régime «concentré », effet négatif du vent sur ceux «avec abri» nourris au même régime.

En revanche, les taurillons du traitement «fourrage sans abri », s'ils ne semblent pas réagir au point de rosée, sont sensibles à la température minimale de la nuit $\left(+3,7\right.$ pulsations $/ \mathrm{mn}$ par $\left.{ }^{\circ} \mathrm{C}\right)$, au rayonnement global $(+8,0$ pulsations $/ \mathrm{mn}$ pour $\left.100 \mathrm{~J} . \mathrm{cm}^{-2} \cdot \mathrm{h}^{-1}\right)$ et à la pluie du jour précédent $(+0,40$ pulsation $/ \mathrm{mn}$ par $\mathrm{mm}$ de pluie).

Le poids vif a un effet positif sur la fréquence cardiaque du traitement «fourrage avec abri »; dans les autres cas, son effet n'est pas significatif.

Le coefficient de détermination des quatre modèles est compris entre 0,36 et 0,48 .

\section{F. Influence de la température du corps noir sur la température rectale et les fréquences respiratoire et cardiaque (tabl. 3)}

La «température du corps noir» n'a pas d'influence significative sur la fréquence cardiaque; elle a une action positive faiblement marquée $\left(+0,028^{\circ} \mathrm{C}\right.$ par ${ }^{\circ} \mathrm{C}$ d'augmentation de $\mathrm{Tb}, \mathrm{P}<0,05)$ sur la température rectale du traitement «fourrage sans abri », mais pas sur celle des autres traitements.

Son effet sur la fréquence respiratoire (tabl. 3) est significatif, sauf pour le traitement «fourrage avec abri »: sans abri, celle-ci augmente respectivement, pour les régimes «concentré » et «fourrage», de 1,5 et $1,2 \mathrm{resp} / \mathrm{mn}$ lorsque $\mathrm{Tb}$ augmente de $1{ }^{\circ} \mathrm{C}$, et avec abri, respectivement de 0,7 et $0,4 \mathrm{resp} / \mathrm{mn}$ par ${ }^{\circ} \mathrm{C}$. 


\section{TableaU 3}

Coefficient de régression et carré du coefficient de corrélation des modèles de régression triple reliant la fréquence respiratoire à la «température du corps noir $»$, alu point de rosée et au poids vif.

Regression coefficients and square of the coefficient of correlation of triple regression models explaining respiratory rhythm by «black body temperature», dewpoint and liveweight.

\begin{tabular}{|c|c|c|c|c|c|c|}
\hline \multicolumn{2}{|c|}{$\begin{array}{c}\text { Fréquence respiratoire } \\
\text { Respiratory rhythm } \\
(\mathrm{resp} / \mathrm{min})\end{array}$} & \multirow{2}{*}{ 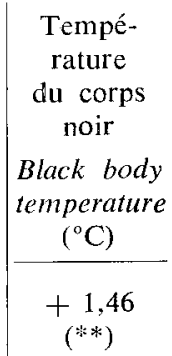 } & \multirow{2}{*}{$\begin{array}{c}\text { Point } \\
\text { de rosée } \\
\begin{array}{c}\text { Dewpoint } \\
\left({ }^{\circ} \mathrm{C}\right)\end{array} \\
\begin{array}{c}+2,34 \\
(*)\end{array}\end{array}$} & \multirow{2}{*}{$\begin{array}{l}\text { Poids vif } \\
\begin{array}{c}\text { Liveweight } \\
\quad(\mathrm{kg})\end{array} \\
\begin{array}{c}0,003 \\
(\mathrm{NS})\end{array}\end{array}$} & \multirow{2}{*}{$\begin{array}{l}\text { Constarte } \\
\text { Constant } \\
-72,5\end{array}$} & \multirow{2}{*}{$\begin{array}{c}\mathbf{R}^{\prime} \\
0,55\end{array}$} \\
\hline Sans abri & $\begin{array}{l}\text { «Concentré» } \\
\text { «Concentrate» }\end{array}$ & & & & & \\
\hline Without shelter & $\begin{array}{c}\text { «Fourrage» } \\
\text { «Forage» }\end{array}$ & $+\underset{(* *)}{1,21}$ & $\begin{array}{l}+0,89 \\
\text { (NS) }\end{array}$ & $\begin{array}{c}-0,104 \\
(*)\end{array}$ & - 9,1 & 0,57 \\
\hline Avec abri & $\begin{array}{l}\text { «Concentré » } \\
\text { «Concentrate» }\end{array}$ & $\begin{array}{c}+0,69 \\
(* *)\end{array}$ & $\begin{array}{l}+0,45 \\
(\mathrm{NS})\end{array}$ & $-_{(* *)}^{0,057}$ & $+12,6$ & 0,56 \\
\hline With shelter & $\begin{array}{c}\text { "Fourrage» } \\
\text { «Forage» }\end{array}$ & $\begin{array}{c}+0 ; 39 \\
(\mathrm{NS})\end{array}$ & $+\underset{(*)}{0,80}$ & $\begin{array}{c}-0,057 \\
(*)\end{array}$ & $+8,3$ & 0,21 \\
\hline
\end{tabular}

$\mathbf{n}=31$ journées d'expérience - 31 experimental days.

(*) $\mathbf{P}<0,05$.

(**) $\mathbf{P}<0,01$.

La figure 1 regroupe, en fonction de la «température du corps noir », les valeurs de la fréquence respiratoire pour l'ensemble des traitements. Les points représentatifs s'ajustent à une courbe polynomiale de $2^{\circ}$ degré, malgré une valeur très élevée de $\mathbf{R r}$ dans un des traitements.

\section{Discussion}

Un fait important ressort de l'étude de la liaison entre les variables physiologiques étudiées et les variables climatiques : seule la fréquence respiratoire est en relation étroite avec ces dernières (tabl. 2), bien que l'étude des moyennes sur la période expérimentale (tabl. 1) ne fasse pas apparaître de différence entre les situations «avec» et «sans abri ». Ce dernier résultat est sans doute dû à la forte variabilité individuelle de la réponse au climat de la fréquence respiratoire, chaque animal suivant les fluctuations climatiques à son propre niveau. 


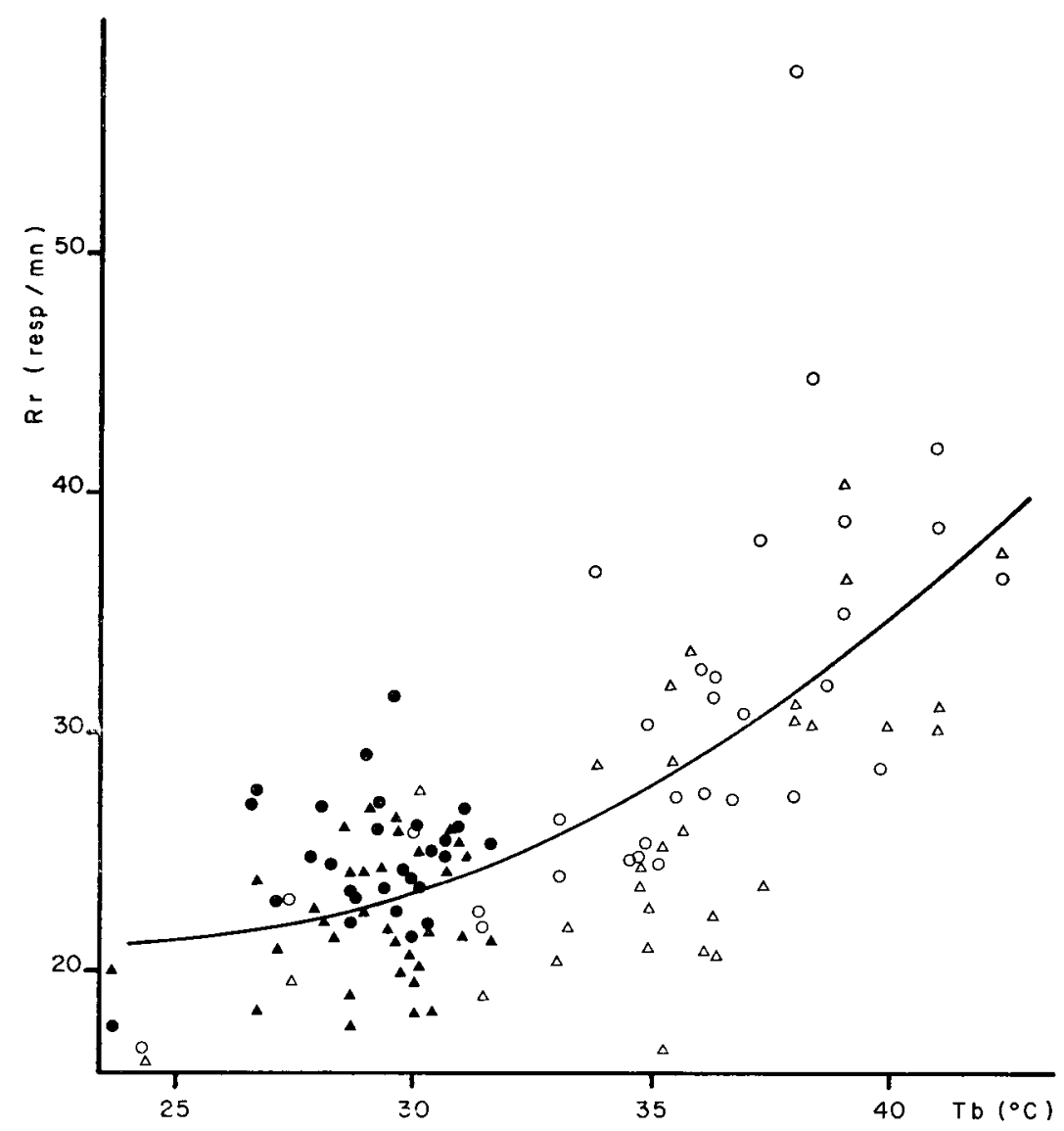

FIG. 1

Fréquence respiratoire (moyenne par traitement de chaque journée d'expérience) en fonction de la température du "corps noir».

Respiratory rhyithm (mean per treatment for each experimental day) as a function of the "black body" temperature.

Ajustement polynomial du $2^{\mathrm{e}}$ degré - $2^{\text {nd }}$ degree polynomial adjustment :

$\mathrm{n}: 124$ donnćes - data.

R : 0,66 .

$\mathbf{Y}: 46.74-2,216 \mathrm{X}+0,048 \mathrm{X}^{2}$.

O Concentré sans abri.

Concentrate without shelter.

$\triangle$ Fourrage sans abri.

Forage without shelter.

- Concentré avec abri.

Concentrate with shelter.

- Fourrage avec abri.

Forage with shelter. 
En revanche, la température rectale, dont la variabilité individuelle est faible, montre un écart significatif entre les deux situations climatiques, alors que même au soleil, son degré de liaison avec les variables climatiques est peu important. La température rectale moyenne des deux lots au soleil ne dépasse que deux fois $39,5^{\circ} \mathrm{C}$; dans les deux cas, ceci est dû au niveau anormal de température d'un seul animal sur les 4 que comporte le lot. On peut donc en conclure que, dans cette étude, les taurillons n'ont pas subi de stress thermique. L'absence d'effet du rayonnement et de Ia température de l'air sur la fréquence cardiaque vient confirmer cette conclusion.

Les taurillons Créoles, placés au soleil, doivent donc équilibrer leur bilan thermique en évacuant de la chaleur par évaporation (notamment respiratoire) mais n'atteignent pas le seuil de stress thermique. Ceci est à rapprocher des résultats (BERBIGIER, 1983 a) relatifs aux performances zootechniques de ces mêmes animaux.

Dans des conditions très semblables, MoraIs \& EsPinosa (1979) observent, à Cuba, sur des femelles Zébu gestantes au pâturage, des valeurs similaires de la température rectale et de la fréquence respiratoire; il en est de même pour AMakiRI \& Funsho (1979) au Nigeria sur des animaux locaux au pâturage. En chambre respiratoire, à une température constante de $31^{\circ} \mathrm{C}$, SEIF, JoHnson \& LippincotT (1979) trouvent, sur des génisses Zébu, une température rectale moyenne de $38,7^{\circ} \mathrm{C}$ et une fréquence respiratoire moyenne de 38 respirations $/ \mathrm{mn}$, peu différentes de nos résultats. La résistance à la chaleur des bovins Créoles semble donc comparable à celle des autres populations bovines tropicales.

Le régime «fourrage» semble provoquer une plus grande sensibilité thermique que le régime «concentré ». Nous avons montré précédemment (BERBIGIER, 1983 a) que les taurillons «sans abri », surtout pour le régime «concentré », réduisent volontairement leur prise de nourriture, sans que leur croissance soit inférieure à ceux du traitement correspondant avec abri. Ceci entraîne une meilleure efficacité alimentaire, et sans doute une moindre dégradation en chaleur de l'énergie des aliments (cf. § III B). De plus, les productions de chaleur estimées sont des moyennes sur la période expérimentale, alors qu'au moment des mesures, l'activité physique d'ingestion doit encore augmenter la production de chaleur des régimes «fourrage», composés de particules d'aliment de grande taille, par rapport aux régimes «concentré » (BouviER, 1977). Ainsi s'explique probablement le fait que, dans une situation climatique donnée, mais particulièrement en l'absence d'abri, les températures rectales des régimes «fourrage 》 soient supérieures à celles des régimes «concentré » (tabl. 1), et que, sans abri, la dépendance des trois variables expliquées par rapport aux variables climatiques soit plus forte pour le régime «fourrage».

Si nous étudions le rôle de chaque variable climatique sur les réponses physiologiques des taurillons nous constatons que :

- sur la fréquence respiratoire s'exerce l'effet du complexe température-point de rosée-rayonnement-vent, dans un sens conforme à l'analyse thermodynamique (BERBIGIER, 1982) : l'augmentation de $\mathrm{Ta}, \mathrm{Tr}, \mathrm{R}$ entraîne une diminution des possibilités de thermolyse, donc une action thermorégulatrice accrue, notamment par l'augmentation des pertes respiratoires, alors que l'augmentation de la vitesse du vent a des conséquences inverses. Il faut noter que le rayonnement ne joue un rôle significatif qu'en l'absence d'abri, ce qui est logique, et pour le régime produisant le plus de chaleur («fourrage»). La température ambiante, dont le rôle est toujours significatif, a une action plus forte en l'absence d'abri, comme le montrent les coefficients de régression correspondants. Il en est de même pour le vent. Les animaux privés d'abri et soumis à un 
rayonnement intense, subissent une contrainte thermique plus forte et réagissent par conséquent ce façon plus marquée à la température et au vent. En revanche, le rôle du point de rosée n'est significatif qu'à l'abri : peut-être la capacité physique d'évaporation de l'interface peau-milieu ambiant, limitée par l'absence de rayonnement et la diminution de la vitesse de l'air, constitue-t-elle alors seulement un facteur limitant, peut-être aussi le fait que les animaux, sous l'abri, aient une partie de leur peau mouillée par leurs déjections augmente-t-il la part de l'évaporation dans la thermolyse totale ;

- sur la température rectale n'apparaît de manière significative que l'effet du rayonnement, en l'absence d'abri et pour le régime «fourrage», comme pour la fréquence respiratoire. Les autres paramètres climatiques n'apparaissent pas, et le pouvo:ir explicatif des modèles est faible;

- sur la fréquence cardiaque, l'action des variables climatiques est de deux types. Pour le traitement «fourrage sans abri », où la contrainte thermique est, comme nous l'avons vu, la plus forte, l'action dominante est celle du complexe températurerayonnement (en l'occurrence la température minimale de la nuit), qui traduit un effet thermodynamique (déséquilibre des échanges de chaleur entre l'animal et le milieu). En revanche, pour les autres traitements, le seul effet ressortant nettement est celui du point de rosée : plus celui-ci est élevé, plus la fréquence cardiaque est rapide. Or, l'animal intégrant l'effet des différentes variables climatiques en un équilibre global thermogénèsethermolyse, l'observation de l'effet isolé d'une seule variable qui, en général, n'a pas le rôle principal, est difficile à expliquer par des considérations physiques et procède sans doute d'un autre ordre de phénomènes.

L'effet positif, constaté en trois occasions, de la pluie du matin ou de la veille sur les fréquences cardiaque et respiratoire, pose un problème : logiquement celle-ci devrait atténuer la contrainte climatique (évaporation à la surface de la peau mouillée). Peut-être l'augmente-t-elle en provoquant une élévation de l'humidité relative de l'air. Mais ici, les possibilités d'artefact sont grancies, car le nombre de jours de pluie est faible (la pluviométrie ne dépasse $5 \mathrm{~mm}$ /jour qu'en 5 occasions sur 44).

L'étude des températures du «corps noir » vient confirmer les conclusions précédentes, bien que la signification des modèles obtenus avec ces dernières soit inférieure à celle des modèles faisant intervenir toutes les variables climatiques : ceci est sans doute partiellement dû au nombre plus réduit de journées d'expérience, mais aussi au fait que le «corps noir» n'intègre pas l'action du climat exactement comme l'animal. Tel quel, le « corps noir » semble néanmoins un bon indicateur de la contrainte climatique subie par les taurillons au soleil et à l'ombre.

\section{Conclusion}

L'ensemble de cette étude montre que les taurillons Créoles, s'ils sont contraints, surtout au soleil, d'augmenter leurs pertes par évaporation pour équilibrer leur bilan de chaleur, ne subissent pas, dans nos conditions expérimentales, de stress thermique proprement dit. Même l'élévation de leur fréquence respiratoire reste modérée. Par ailleurs nous avons montré que leur croissance n'était pas perturbée par l'exposition au soleil (Berbigier, 1983 a). Enfin, les niveaux de température rectale et de fréquence respiratoire observés sont comparables à ceux d'autres races tropicales dans des 
conditions climatiques similaires. L'augmentation importante de la contrainte thermique due à l'exposition au soleil a donc peu d'effet sur les taurillons Créoles. Ces animaux paraissant bien adaptés à leur milieu thermique, il semble intéressant de chercher à améliorer leurs performances par sélection ou par croisement avec d'autres races bovines issues des milieux tempérés.

Accepté pour publication en juin 1983.

\title{
Remerciements
}

L'auteur remercie M. Armand Grude, Directeur du Domaine I.N.R.A. de Gardel, et M. Dumaine Duchant sans lesquels cette expérience n'aurait pas pu être réalisée.

\author{
Summary \\ Effect of humid tropical climate on rectal temperature and respiratory \\ and cardiac rhythms of Creole young bulls in Guadeloupe \\ (French West Indies)
}

\begin{abstract}
In order to study the effect of tropical climate on some physiological parameters, eleven Creole young bulls were subjected to two climatic treatments (in the open air without shelter and in an open stable with shelter) and to two dietary treatments (a «concentrate» diet composed of 80 p. 100 concentrate and 20 p. 100 forage and a "forage» diet composed of 32 p. 100 concentrate and 68 p. 100 forage) according to a $2^{2}$ factorial design. Between 11 a.m. and 1 p.m. rectal temperature, respiratory and cardiac rhythms, climatic variables and "black body" temperature $(10 \mathrm{~cm}$ diameter black globe) were measured during the 44 days of the experiment. The effects of these treatments were studied using the analysis of variance, the dependence upon climatic variables by means of multiple regression analysis.
\end{abstract}

Respiratory rhythm was the most dependent on the climatic variables, though the ditrerence between the climatic treatments (with and without shelter) was not significant. Rectal temperature for which this difference reached the $\mathrm{P}<0.01$ level, was nevertheless little dependent on climatic variables, like cardiac rhythm (tabl. 1 and 2). In all the cases the effect of climate was stronger without shelter and particularly with the «forage» diet which produced more heat than the «concentrate».

"Black body» temperature was found to be a good index of the climatic «pressure» exerted on the animals (tabl. 3 and fig. 1).

This study showed that Creole young bulls had to increase thermolysis by evaporation to equilibrate their heat balance. Nevertheless, they were not thermally stressed (rectal temperature remained moderate). They seem well adapted to the West Indian tropical climate.

\section{Références bibliographiques}

AMAKIRI S.F., Funsho O.N., 1979. Studies of rectal temperature, respiratory rate and heat tolerance in cattle in the humid tropics. Anim. Prod., 28, 329-335.

Berbigier P., 1982. Analyse physique des pertes thermiques de l'animal vers son environnement. Influence des facteurs climatiques, 65-96. In : Actions du climat sur l'animal au pâturage, I.N.R.A. Publ., Versailles. 
Berbigier P., 1983 a. Effet du climat tropical humide sur la consommation d'aliment et d'eau et sur la vitesse de croissance de taurillons Créoles en Guadeloupe. Ann. Zootech., 32, 93-108.

Berbigier P., 1983 b. Tolérance au climat tropical des taurillons Frisons et Créoles soumis à plusieurs régimes alimentaires. Détermination d'un indice climatique. Ann. Zootech., 32, 383-396.

Bouvier J.C., 1977. Etude descriptive des cinétiques des échanges gazeux du mouton. Influence de la ratio, du niveau d'alimentation et des phénomènes digestifs. Thèse de Docteur-Ingénieur, Université de Clermont II, $124 \mathrm{p}$.

Dagnelie P. Théorie et méthodes statistiques, tome 2, 463 p. Les Presses Agronomiques, Gembloux.

INGRAM D.L., MounT L.E., 1975. Man and animals in hot environments, 185 p. SpringerVerlag, Berlin - Heidelberg - New York.

I.N.R.A., 1978. Alimentation des ruminants, 47-88. I.N.R.A. Publ., Versailles.

Morais M., EsPinOSA J., 1979. Efecto de la temperatura ambiental y la humedad relativa sobre la temperatura rectal y la frecuencia respiratoria en vacas cebù no lactantes. Rev. Sal. Anim., 1, 83-88.

Mount L.E., 1974. The concept of thermal neutrality, 426-439. In : Monteith J.L., MounT L.E., Heat loss from animals and man, Butterworths, London.

Seif S.M., Johnson H.P., Lippincott A.C., 1979. The effects of heat exposure $\left(31{ }^{\circ} \mathrm{C}\right)$ on Zebu and Scottish Highland Cattle. Int. J. Biometeorol., 23, 9-14.

SNedecor G.W., Cochran W., 1971. Méthodes statistiques, 649 p. Association de coordination technique agricole, Paris (édition française). 